\title{
Recent experiments in inverse kinematics with the magnetic spectrometer PRISMA
}

E. Fioretto ${ }^{1}$, L. Corradi ${ }^{1}$, D. Montanari ${ }^{2}$, S. Szilner $^{3}$, G. Pollarolo ${ }^{4}$, F. Galtarossa ${ }^{1,5}$, D. Ackermann 6 , G. Montagnoli ${ }^{7}$, F. Scarlassara ${ }^{7}$, A.M. Stefanini ${ }^{1}$, S. Courtin $^{2}$, A. Goasduff ${ }^{1}$, F. HaAs $^{2}$, D. Jelavić-Malenica ${ }^{3}$, C. Michelagnoli ${ }^{8}$, T. Mijatović ${ }^{3}$, N. Soić ${ }^{3}$, C. UR $^{9}$ and J.J. VALIENTE-DobON ${ }^{1}$

${ }^{1}$ INFN, Laboratori Nazionali di Legnaro, Legnaro (PD), Italy ${ }^{2}$ IPHC, CNRS-IN2P3 and Université de Strasbourg, Strasbourg, France

${ }^{3}$ Ruđer Bošković Institute, Zagreb, Croatia

${ }^{4}$ Dip. di Fisica Teorica, Università di Torino and INFN, Torino, Italy

${ }^{5}$ Dip. di Fisica e Scienze della Terra, Università di Ferrara, Ferrara, Italy ${ }^{6}$ GSI, Darmstadt, Germany

${ }^{7}$ Dip. di Fisica e Astronomia, Univ. di Padova and INFN, Padova, Italy ${ }^{8}$ GANIL, CEA/DSM-CNRS/IN2P3, Caen Cedex 05, France

${ }^{9}$ Horia Hulubei National Institute of Physics and Nuclear Engineering, Bucharest, Romania

\begin{abstract}
In the last period, two classes of experiments have been carried out with the large acceptance magnetic spectrometer PRISMA. In particular, the one- and two-neutron transfer processes at energies ranging from the Coulomb barrier to deep below it and the population of exotic neutron rich nuclei in the $A \sim 130$ and $A \sim 200$ mass regions have been studied. Both kinds of experiments have been performed in inverse kinematics identifying in $A, Z$ and velocity the light target-like recoils with PRISMA placed at very forward angles in order to have, at the same time, high efficiency and good energy and mass resolutions.
\end{abstract}




\section{Introduction}

Recent studies on transfer processes benefited from the construction of new generation tracking spectrometers, based on the trajectory reconstruction, and the use of the state-of-art large area particle detectors. The large acceptances of these spectrometers and the high resolving powers of their detection systems allowed to investigate the transfer process around and well below the Coulomb barrier with high efficiency and good fragment identification.

In this work we report on studies on the interplay between single and pair transfer modes at near- and sub-barrier energies and on the population of neutron rich nuclei in the $A \sim 130$ and $A \sim 200$ mass regions around the Coulomb barrier. The measurements have been performed in a very efficient way making use of inverse kinematics reactions and the large solid angle magnetic spectrometer PRISMA [1-3] installed at Laboratori Nazionali di Legnaro.

\section{Sub-barrier transfer measurements}

In the sub-barrier region interacting nuclei are only slightly influenced by the nuclear potential and follow almost pure Coulomb trajectories. Therefore, only few transfer channels are open, excitation energies are restricted to few $\mathrm{MeV}$ and uncertainties in calculations associated with optical potentials can be minimized [4]. Available data on transfer reactions in this energy range are extremely scarce because of various kinds of significant experimental difficulties which can be overcome making use of inverse kinematics detecting the lighter target-like fragments with magnetic spectrometers at very forward angles $[5,6]$.

Transfer processes were investigated at LNL in closed shell nuclei (or nearly so) for both neutrons and protons with the ${ }^{96} \mathrm{Zr}+{ }^{40} \mathrm{Ca}$ reaction [7] and in superfluid nuclei with the ${ }^{116} \mathrm{Sn}+{ }^{60} \mathrm{Ni}$ system [8]. The ${ }^{96} \mathrm{Zr}$ and ${ }^{116} \mathrm{Sn}$ beams were delivered by the Tandem/PIAVE-ALPI accelerator complex at energies ranging from the Coulomb barrier down to $25 \%$ below with an average current of about $2 \mathrm{pnA}$. Light target-like ${ }^{40} \mathrm{Ca}$ and ${ }^{60} \mathrm{Ni}$ ions were detected at a very forward angle $\left(\theta_{l a b}=20^{\circ}\right)$ with the large acceptance magnetic spectrometer PRISMA.

Excitation functions of the main transfer channels were measured and yields were detected at the level of $10^{-4}$ with respect to the elastic channel. In fig. 1 we show an example of two-dimensional plot velocity (reconstructed via time-of-flight) vs in-plane scattering angle for the ${ }^{96} \mathrm{Zr}+{ }^{40} \mathrm{Ca}$ reaction at 


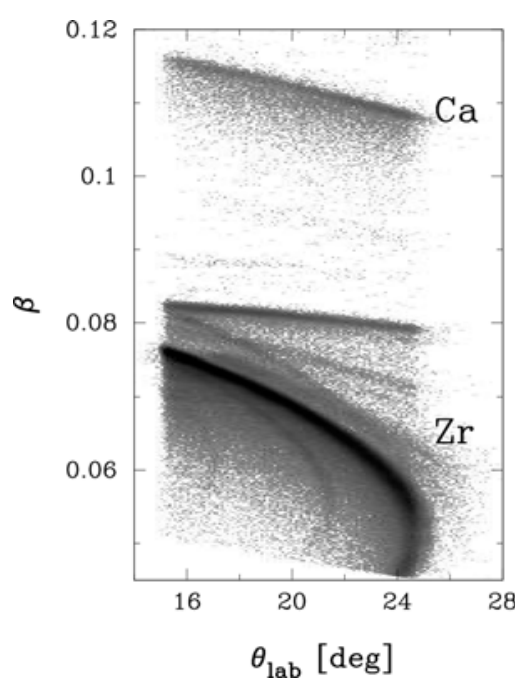

Figure 1: Velocity $(\beta=\mathrm{v} / \mathrm{c})$ vs inplane scattering angle $\left(\theta_{\text {lab }}\right)$ for the reaction ${ }^{96} \mathrm{Zr}+{ }^{40} \mathrm{Ca}$ at $E_{l a b}=330$ $\mathrm{MeV}$.
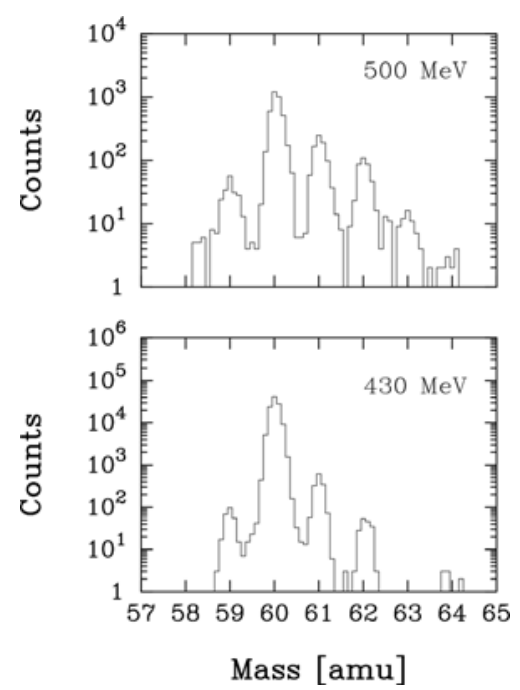

Figure 2: Mass spectra of Ni isotopes in the ${ }^{116} \mathrm{Sn}+{ }^{60} \mathrm{Ni}$ system at two representative energies, above and below the Coulomb barrier.

$E_{l a b}=330 \mathrm{MeV}$. One sees the events corresponding to Ca-like recoils as well as those corresponding to Zr-like ions entering into PRISMA. The ridges of these Zr-like ions are due to scattering on $\mathrm{Ca}$ (main ridge presenting a bending around the kinematic limiting angle $\left(\theta_{l a b}=24.6^{\circ}\right)$ ), on lighter elements (with smaller limiting angles) and on other heavier elements (target contaminants). The almost horizontal ridge, with high yield and with a $\beta$ $\sim 0.08$, is due to $\mathrm{Zr}$ scattering in direct kinematics. The very good mass resolution $\Delta A / A \sim 1 / 240$ is guaranteed by the high kinetic energy of the recoils due to the inverse kinematics. As an example, mass spectra for $\mathrm{Ni}$ isotopes, measured for ${ }^{116} \mathrm{Sn}+{ }^{60} \mathrm{Ni}$ reaction at two bombarding energies, are displayed in fig. 2 .

The experimental transfer probabilities as a function of the distance of closest approach for the $(+1 n)$ and $(+2 n)$ neutron transfer channels have been compared with results of microscopic calculations. The theoretical two-neutron transfer probabilities have been computed including nucleonnucleon correlations (see Ref $[7,8]$ for details). For the first time with heavy ions, experimental transfer probabilities have been well reproduced (in absolute values and in slope) by microscopic calculations which include nucleonnucleon correlations. 


\section{Population of heavy neutron rich nuclei}

Certain neutron rich regions of the nuclide chart, like those below ${ }^{208} \mathrm{~Pb}$ or in the actinides and transactinides, can be hardly accessed by fragmentation or fission reactions, and multinucleon transfer represents a suitable mechanism to approach these areas. We remind that nuclear properties of neutron rich nuclei around $\mathrm{N}=126$, relevant for r-process, play a critical role for theoretical predictions of the synthesis of the heaviest elements [9], for disentangling a variety of astrophysical scenarios $[10,11]$ and to study the competition between Gamow-Teller and First-Forbidden $\beta$ transitions [11].

It is worthwhile to mention the experiment very recently carried out in GANIL using the large acceptance magnetic spectrometer VAMOS coupled to the $\gamma$-array EXOGAM. Its main goal was to measure the yield distribution of light and associated heavy transfer products populated in the ${ }^{136} \mathrm{Xe}+{ }^{198} \mathrm{Pt}$ reaction [12] at an energy of about $8 \mathrm{MeV} / A$. Another (integral) experiment was performed at Jyväskylä using a gas cell system to extract and study, offline by means of $\beta-\gamma$ coincidences, nuclei in the Os region populated through the reaction ${ }^{136} \mathrm{Xe}+{ }^{208} \mathrm{~Pb}$ in deep inelastic processes. Both experiments were carried out during the preparatory phases of the facilities KISS (KEK Isotope Separator System) at RIKEN and GaLS (Gas cell and Laser ion Separation) at the Flerov Laboratory in Dubna [13] aiming to the study of $\beta$-decay spectroscopy of heavy neutron rich nuclei in the $\mathrm{A} \sim 200$ and $\mathrm{N} \sim 126$ region populated via deep inelastic reactions.

Within this context, an experiment was recently carried out at LNL in which the yields of light and heavy fragments were measured by using the kinematic coincidence technique. To this end the spectrometer (the main arm of the coincidence) was coupled to a second arm (for the direct detection of heavy partner) which consists of a position sensitive Parallel Plate Avalanche Counter (PPAC) followed by an axial field ionization chamber (IC). In order to overcome the experimental difficulties related to the direct detection of the heavy partner, multineutron and multiproton transfer channels were populated via the inverse kinematics reaction ${ }^{197} \mathrm{Au}+{ }^{130} \mathrm{Te}$ at $E_{l a b}=1070 \mathrm{MeV}$. The ${ }^{197} \mathrm{Au}$ beam, delivered by PIAVE-ALPI accelerator complex with an intensity of $2 \mathrm{pnA}$, represents the presently heaviest beam available at LNL. The PRISMA spectrometer was placed around the grazing angle $\left(\theta_{l a b}=+37^{\circ}\right)$ with respect to the beam axis in order to identify the lighter target-like fragments populated in the reaction. Projectile-like fragments were detected with the second arm placed at about $90 \mathrm{~cm}$ of distance from the target and at the kinematically correlated angle $\theta_{l a b}=-37^{\circ}$. 


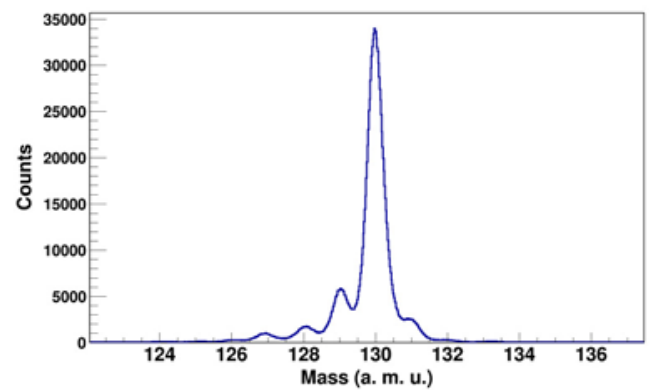

Figure 3: Mass distribution of tellurium isotopes at the focal plane of PRISMA measured in the ${ }^{197} \mathrm{Au}+{ }^{130} \mathrm{Te}$ reaction at $E_{l a b}=1070 \mathrm{MeV}$ and $\theta_{l a b}=+37^{\circ}$.

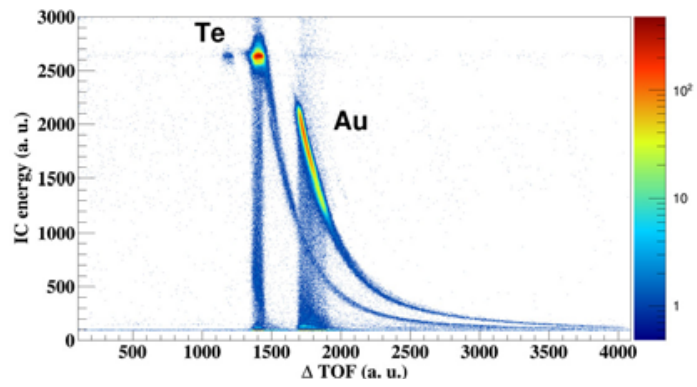

Figure 4: Energy vs $\Delta$ TOF spectrum for fragments produced in the ${ }^{197} \mathrm{Au}+{ }^{130} \mathrm{Te}$ reaction at $E_{l a b}=1070 \mathrm{MeV}$ (see text for details).

The main goal of the measurement was to study the population of neutron rich nuclei in the $A \sim 130$ and $A \sim 200$ mass regions. In particular, via the two-proton stripping $(-2 p)$ and four-neutron pick-up $(+4 n)$ channel one should be able to populate ${ }^{132} \mathrm{Sn}$, which represents a benchmark neutron rich nucleus for different physics cases, reported in the proposals of forthcoming radioactive beam facilities. In addition, proton pick-up and neutron stripping channels from ${ }^{130}$ Te would lead, from the heavy partner side, to the population of neutron rich nuclei in the Pt-Os region of astrophysics interest. Secondary processes should play a non negligible role for these heavy nuclei, thus it will be interesting to compare the final yields with those expected from theoretical models, already successfully applied for lower mass systems.

The PRISMA spectrometer was set for the selection and identification of the target-like fragments thus measuring $A, Z$ and $Q$-value distributions for the light partner. As a preliminary result, the yields of tellurium isotopes measured with PRISMA are shown in fig. 3. Using PRISMA events as trigger of the DAQ, coincident fragments were detected in the second 
arm. As additional parameter for the fragment discrimination, we used the difference $\triangle \mathrm{TOF}$ of the time-of-flight of the fragments measured between the start detector of PRISMA and the PPAC of the second arm, respectively. Typical Energy (measured with IC) vs $\triangle$ TOF scatter plot is shown in fig. 4 where one can observe a clear separation between projectile-like and target-like fragments.

The data analysis for the heavy partner is still in progress from which we expect to get important information on the production yield of heavy neutron rich nuclei and to identify any significant contribution from the onset of secondary processes such as the transfer induced fission.

\section{Acknowledgement}

This work was partly supported by European Union Seventh Framework Programme FP7/2007-2013 under Grant No. 262010 - ENSAR.

\section{References}

[1] A.M. Stefanini et al., Nucl. Phys. A 701, 217c (2002).

[2] S. Szilner et al., Phys. Rev. C 76, 024604 (2007).

[3] L. Corradi et al., Nucl. Instr. and Meth. in Phys. Res. B 317, 743 (2013).

[4] L. Corradi, G. Pollarolo, and S. Szilner, J. of Phys. G 36, 113101 (2009).

[5] C.L. Jiang et al., Phys. Lett. B 337, 59 (1994).

[6] C.L. Jiang et al., Phys. Rev. C 57, 2393 (1998).

[7] L. Corradi et al., Phys. Rev. C 84, 034603 (2011).

[8] D. Montanari et al., Phys. Rev. Lett. 113, 052501 (2014).

[9] V. Zagrebaev and W. Greiner, Phys. Rev. Lett. 101, 122701 (2008).

[10] K.-L. Kratz et al., Astrophys. J. 403, 216 (1994).

[11] H. Grawe et al., Rep. Prog. Phys. 70, 1525 (2007).

[12] Y.X. Watanabe et al., Phys. Rev. Lett., accepted (2015).

[13] K. Novikov et al., J. of Phys.: C.S. 515, 012016 (2014). 